\title{
CONTROL OF A HEAT EXCHANGER USING NEURAL NETWORK PREDICTIVE CONTROLLER COMBINED WITH AUXILIARY FUZZY CONTROLLER
}

\author{
Neethu T R ${ }^{1}$, Kalaichelvi $\mathrm{P}^{2}$, Vetriselvi $\mathrm{V}^{3}$ \\ ${ }^{1,2}$ Department of Chemical Engineering, \\ National Institute of Technology Tiruchirappalli, \\ Tiruchirappalli, 620015, Tamilnadu, India, \\ ${ }^{3}$ Department of ICE, National Institute of Technology Tiruchirappalli, \\ Tiruchirappalli, 620015 Tamilnadu, India.
}

\begin{abstract}
The paper presents an advanced control strategy that uses the neural network predictive controller and the fuzzy controller in the complex control structure with an auxiliary manipulated variable. The controlled tubular heat exchanger is used for pre-heating of petroleum by hot water. The heat exchanger is modelled as a nonlinear system with the interval parametric uncertainty. The set point tracking and the disturbance rejection using intelligent control strategies are investigated. The control objective is to keep the outlet temperature of the pre-heated petroleum at a reference value. Simulations of control of the tubular heat exchanger are done in the Matlab/Stimulant environment. The complex control structure with two controllers is compared with the conventional PID control, fuzzy control and NNPC. Simulation results confirm the effectiveness and superiority of the complex control structure combining the NNPC with the auxiliary fuzzy controller.
\end{abstract}

\section{KEYWORDS}

MPC, NNPC, NNMPC, System identification, Control of plate heat exchanger

\section{INTRODUCTION}

Model Predictive Control (MPC), a control algorithm which uses an optimizer to solve for the optimal control moves over a future time horizon based upon a model of the process, has become a standard control technique in the process industries over the past two decades. In most industrial applications, a linear dynamic model developed using empirical data is used even though the process itself is often nonlinear. Linear models have been used because of the difficulty in developing a generic nonlinear model from empirical data and the computational expense often involved in using nonlinear models. In this paper, we present a generic neural network based technique for developing nonlinear dynamic models from empirical data and show that these models can be efficiently used in a model predictive control framework. This nonlinear MPC 
International Journal of Instrumentation and Control Systems (IJICS) Vol.8, No.2, April 2018

based approach has been successfully implemented in a number of industrial applications in the refining, petrochemical, paper and food industries. Performance of the controller on a nonlinear industrial process, a polyethylene reactor, is presented.

Model Predictive Control (MPC), a control calculation which utilizes an optimizer to unravel for the optimal control moves over a future time horizon based upon a model of the process, has turned into a standard control strategy in the process commercial ventures in the course of recent decades.

NN have been shown to have good approximation capability for non-linear systems. A large number of predictive control schemes have been developed based on Multi Layer Perception (MLP) neural network models since 1990.The key to the successful application of non-linear predictive controller based on a neural network model is an accurate nonlinear model and an efficient optimization algorithm. The back propagation learning algorithm, commonly used in MLP, is essentially a non-linear steepest descent algorithm. The aim of controller design is to construct a controller that generates control signals that in turn generate the desired plant output subject to given constraints. Predictive control tries to predict, what would happen to the plant output for a given control signal. In this way, we know in advance, what effect the control will have, and by this knowledge the best possible control signal is chosen.

\section{SYSTEM IDENTIFICATION}

The first stage of model predictive control is to train a neural network to represent the forward dynamics of the plant. The prediction error between the plant output and the neural network output is used as the neural network training signal. The process is represented by Fig.1.

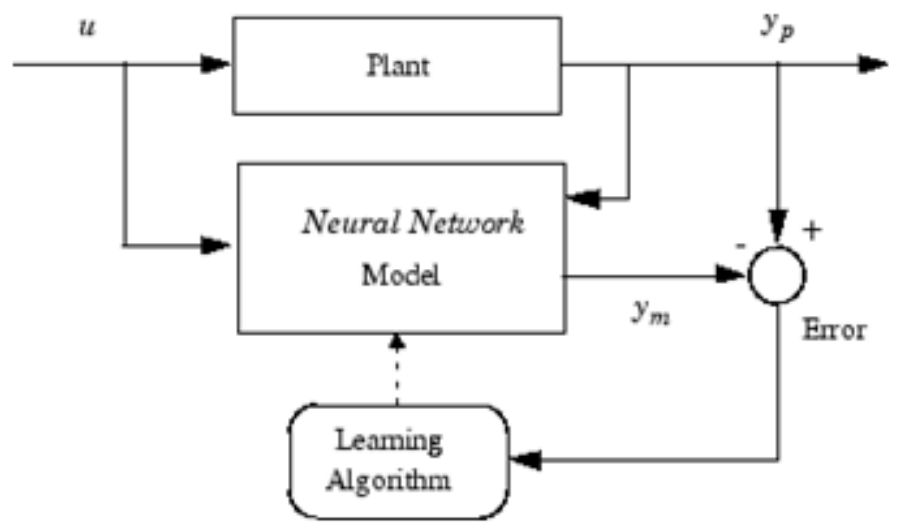

Fig.1 Training of Neural Network

The neural network plant model uses previous inputs and previous plant outputs to predict future values of the plant output. The structure of the neural network plant model is given in Fig.2.

This network can be trained offline in batch mode, using data collected from the operation of the plant. We can use any of the training algorithms for network training. This process is discussed in more detail in following sections. 


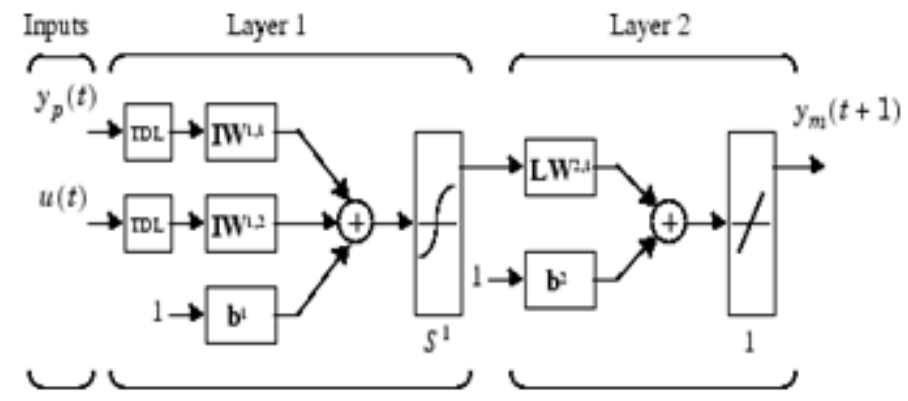

Fig.2 Structure of Neural Network Plant Model

\section{PREDiCTIVE CONTROL}

The model predictive control method is based on the receding horizon technique. The neural network model predicts the plant response over a specified time horizon. The predictions are used by a numerical optimization program to determine the control signal that minimizes the following performance criterion over the specified horizon.

$$
J=\sum_{j=N_{1}}^{N_{2}}(y r(t+j)-y m(t+j))^{2}+\sum_{j=1}^{N_{u}}\left(u^{\prime}(+j-1)-u^{\prime}(t+j-2)\right)^{2}
$$

where $N_{1}, N_{2}$, and $N_{u}$ define the horizons over which the tracking error and the control increments are evaluated. The $u^{\prime}$ variable is the tentative control signal, $y_{r}$ is the desired response, and $y_{m}$ is the network model response. The $\rho$ value determines the contribution that the sum of the squares of the control increments has on the performance index.

Fig.3 illustrates the neural network predictive control process. The controller consists of the neural network plant model and the optimization block.

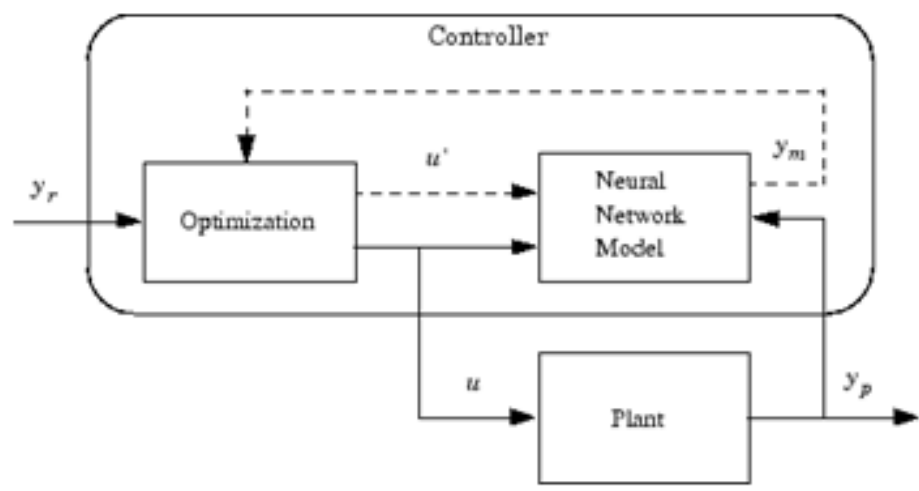

Fig.3 Illustration of Neural Network Predictive Control Process

The optimization block determines the values of $u^{\prime}$ that minimize $J$, and then the optimal $u$ is input to the plant. The program generates training data by applying a series of random step inputs to the Simulink plant model as shown in Fig.4. 
International Journal of Instrumentation and Control Systems (IJICS) Vol.8, No.2, April 2018

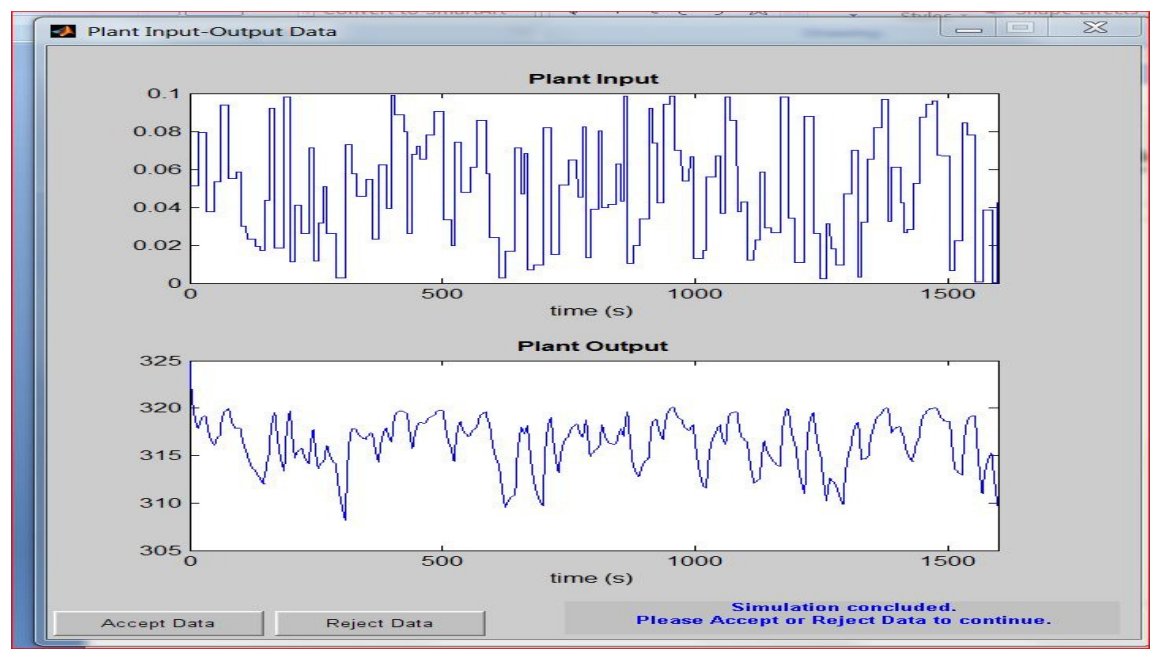

Fig. 4 Plant Input Output Data's for Training

\section{Control of Plate Heat Exchanger Using Neural Network}

The neural network predictive controller that is implemented in the Neural Network Toolbox software uses a neural network model of a nonlinear plant to predict future plant performance. The controller then calculates the control input that will optimize plant performance over a specified future time horizon. The objective of the controller is to maintain the outlet temperature of cold fluid by adjusting the mass flow rate of hot fluid $\mathrm{m}_{\mathrm{h}}$. Also keep the mass flow rate of cold fluid as $\mathrm{m}_{\mathrm{c}}=0.0112 \mathrm{~kg} / \mathrm{s}$.

The first step in neural network plant model predictive control is to determine the neural network plant model. For that first we have to find out the mathematical plant model of plate heat exchanger, based on the energy balance equation of plate heat exchanger. Unsteady-state energy balances have been used as the basis for the derivation of the mathematical model for the plate heat exchanger. Assuming $U$ to be constant, the unsteady-state energy balance around the cold plate is given by:

$$
m_{c} C_{p}\left(T_{c i}-T_{c o}(t)\right)+m_{h}(t) C_{p}\left(T_{h i}-T_{h o}(t)\right)=M_{c} C_{p} \frac{d T_{c o}(t)}{d t}
$$

And the unsteady-state energy balance around the hot plate is given by:

$$
\mathrm{m}_{\mathrm{h}} \mathrm{C}_{\mathrm{p}}\left(\mathrm{T}_{\mathrm{hi}}-\mathrm{T}_{\mathrm{ho}}(\mathrm{t})\right)+\mathrm{m}_{\mathrm{c}}(\mathrm{t}) \mathrm{C}_{\mathrm{p}}\left(\mathrm{T}_{\mathrm{ci}}-\mathrm{T}_{\mathrm{co}}(\mathrm{t})\right)=\mathrm{M}_{\mathrm{h}} \mathrm{C}_{\mathrm{p}} \frac{\mathrm{dT} \text { ho }(\mathrm{t})}{\mathrm{dt}}
$$

Simulink diagram of plate heat exchanger has drawn in Simulink editor based on the energy balance equation of plate heat exchanger is shown in Fig. 5.

The neural model has been trained using data set obtained from dynamic equations of plate heat exchanger. The feed forward network with sigmoidal activation function was chosen based on the trials with different structures of multilayer perception. The lowest error corresponds to 8 neurons in the hidden layer. Hence it was selected as optimal architecture of ANN. The ANN selected here consists of 4 neurons in the input layer, 7 neurons in the hidden layer and one neuron in the output layer. The training algorithm used in this modelling is multi-layer perception algorithm. 


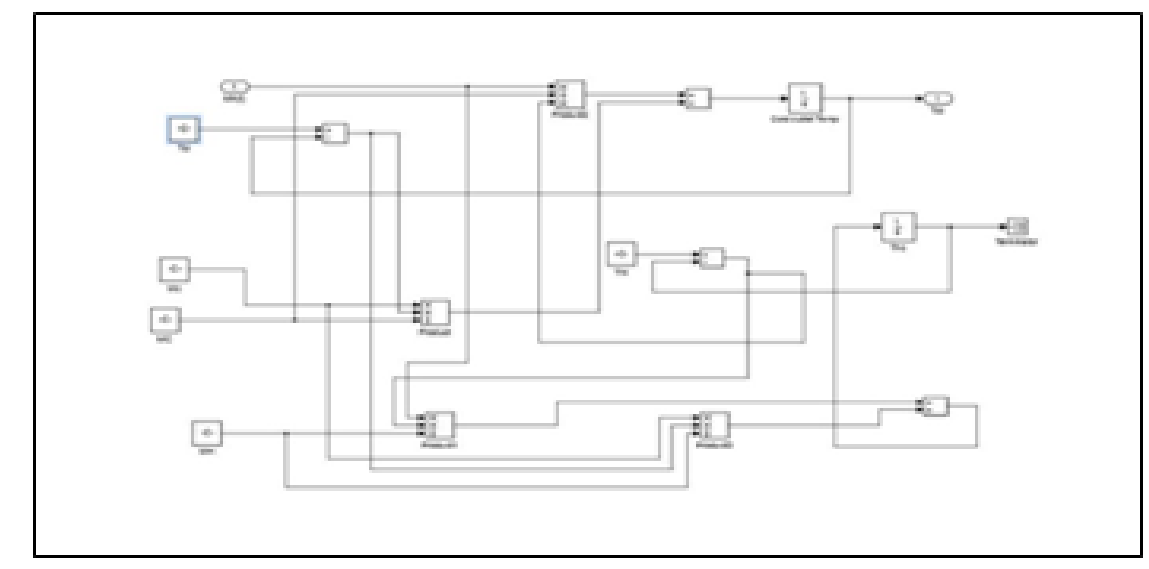

Fig. 5 Simulink Diagram of Energy Balance Equation of PHE

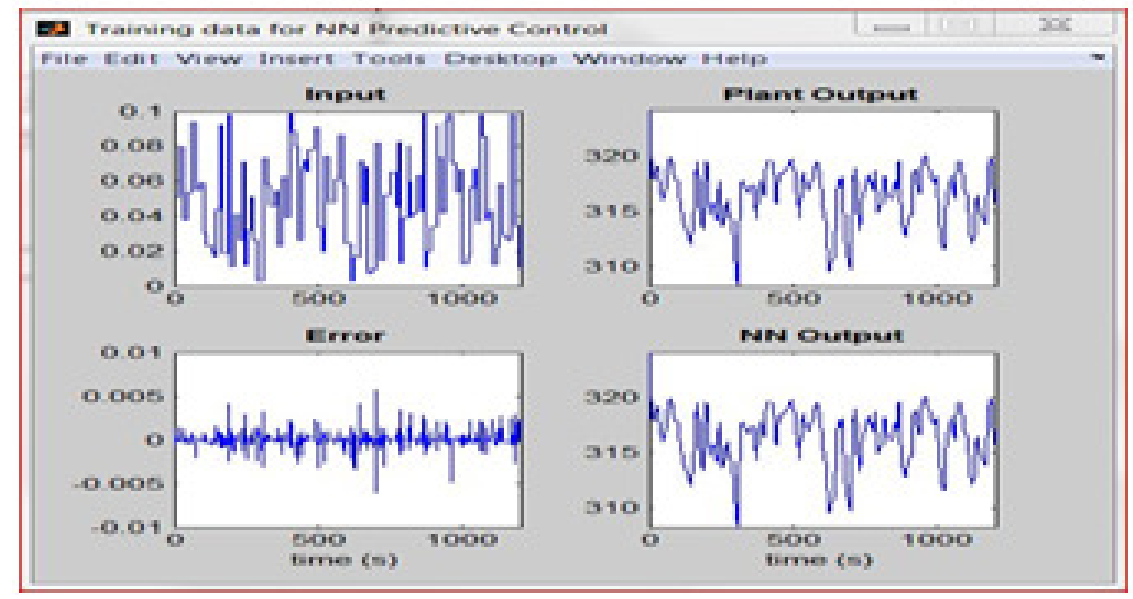

Fig. 6 Response of Plant Model after Training

The response of plant model after training is complete is shown in Fig.6. The error shown in Fig. 6 is the Prediction error which is the difference between the plant output and the output of the neural network plant model. This prediction error is used for the training of the neural network. Neural Network plant model generates the control signal which actually is one step ahead the prediction of the controller which is depicted as NN output in Fig.6. Neural network predictive controller has designed by varying controller horizons $\mathrm{N}_{2}$ and $\mathrm{N}_{\mathrm{u}}$, control weighting factor $\rho$, search parameter $\alpha$. The weighting parameter $\rho$, it multiplies the sum of squared control increments in the performance function. The parameter $\alpha$ is used to control the optimization. It determines how much reduction in performance is required for a successful optimization step. We can select which linear minimization routine is used by the optimization algorithm, and we can decide how many iterations of the optimization algorithm are performed at each sample time. The values of controller parameters have chosen for neural network predictive control is shown in Figure 1.1. 
International Journal of Instrumentation and Control Systems (IJICS) Vol.8, No.2, April 2018

Table 1.1 Neural Network Predictive Control Parameters

\begin{tabular}{|l|l|}
\hline Controller parameters & Values \\
\hline Cost horizon & 8 \\
\hline Control horizon & 4 \\
\hline Control weighing factor & 0.05 \\
\hline Search parameter & 0.001 \\
\hline
\end{tabular}

The designed controller uses a neural network model to predict future plate heat exchanger responses to potential control signals.

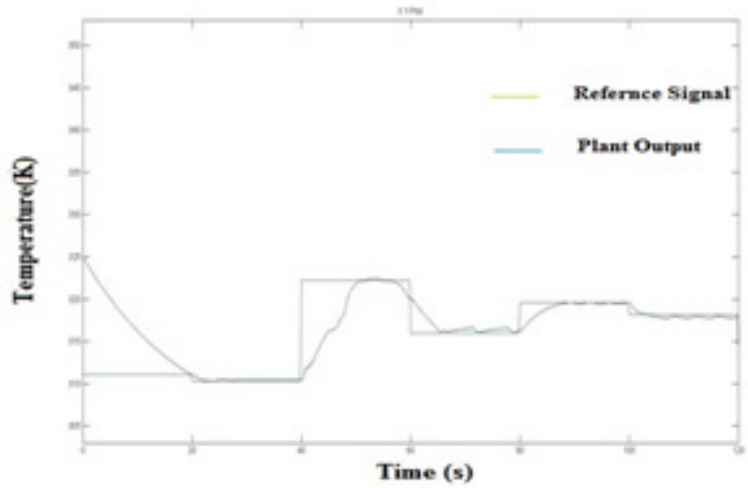

Fig. 1.6 Plant Output and Reference Signal

Fig. 1.6 shows the plant output and reference signal. The result obtained for the random reference signal proved the tracking ability of controller. Also almost offset free and very close set point tracking was obtained using NN predictive control strategy. So from the graph, it can be seen that the neural network predictive controller strategy successfully tracks the random reference signal.

\section{CONCLUSION}

The result obtained for the random reference signal illustrates and proves the tracking ability of controller. Also almost offset free and very close set point tracking is obtained using NNMPC strategy.

In this paper the consideration of dynamic neural models in predictive control for a benchmark nonlinear process, plate heat exchanger is presented. Neural network controller was used to maintain the outlet temperature of cold solution (Xanthum gum solution) by adjusting the mass flow rate of hot water. Non linear auto regressive with exogenous input was recognized utilizing MLP, and approved on the data produced from the simulation of plate heat exchanger dynamic equations. This model represents the dynamics of the nonlinear plate heat exchanger and is utilized as nonlinear predictor in the neural network predictive controller. On analysis of the response graph (Figure 7.6), it can be seen that the neural network predictive controller strategy successfully tracks the random reference signal. The outcome got for the random reference signal outlines and demonstrates the tracking ability of controller. Using the NNMPC strategy almost offset free and close set point tracking is obtained. 
International Journal of Instrumentation and Control Systems (IJICS) Vol.8, No.2, April 2018

\section{REFERENCES}

[1] R.W. Serth, T.G. Lestina, 3 e Heat Exchangers, Process Heat Transfer, in: Principles, Applications and Rules of Thumb, 2014, pp.

[2] F. Peng, G. Cui, Efficient simultaneous synthesis for heat exchanger network with simulated annealing algorithm, Appl. Therm. Eng. 78 (2015) .

[3] J.J. Klemes, P.S. Varbanov, Heat integration including heat exchangers, combined heat and power, heat pumps, separation processes and process control, Appl. Therm. Eng. 43 (2012).

[4] T.G. Walmsley, M.R.W. Walmsley, A.S. Morrison, M.J. Atkins, J.R. Neale, A derivative based method for cost optimal area allocation in heat exchanger networks, Appl. Therm. Eng. 70 (2) (2014).

[5] A. Preglej, J. Rehrl, D. Schwingshackl, I. Steiner, M. Horn, I. Skrjanc, Energyefficient fuzzy modelbased multivariable predictive control of a HVAC system, Energy Build. 82 (2014).

[6] R.F. Garcia, Improving heat exchanger supervision using neural networks and rule based techniques, Expert Syst. Appl. 39 (3) (2012).

[7] T.A. Tahseen, M. Ishak, M.M. Rahman, Performance predictions of laminar heat transfer and pressure drop in an in-line flat tube bundle using an adaptive neuro-fuzzy inference system (ANFIS) model, Int. Commun. Heat Mass Transf. 50 (2014).

[8] J.A. Hernandeza, D. Coloradoa, O. Cort es-Aburtob, Y. El Hamzaouia, V. Velazqueza, B. Alonsoa, Inverse neural network for optimal performance in polygeneration systems, Appl. Therm. Eng. 50 (2) (2013).

[9] R.E. Precup, H. Hellendoorn, A survey on industrial applications of fuzzy control, Comput. Ind. 62 (3) (2011).

[10] I. Babuska, R.S. Silva, Dealing with uncertainties in engineering problems using only available data, Comput. Methods Appl. Mech. Eng. 270. 